\title{
Quantification of myocardial strain assessed by cardiovascular magnetic resonance feature tracking in healthy subjects-influence of segmentation and analysis software
}

\author{
Carolin Lim ${ }^{1,2} \cdot$ Edyta Blaszczyk ${ }^{1,2}$ - Leili Riazy ${ }^{1,2,3} \cdot$ Stephanie Wiesemann ${ }^{1,2}$ • Johannes Schüler ${ }^{1}$. \\ Florian von Knobelsdorff-Brenkenhoff ${ }^{1,2,4}$. Jeanette Schulz-Menger ${ }^{1,2}$
}

Received: 4 April 2020 / Revised: 16 September 2020 / Accepted: 17 November 2020 / Published online: 4 December 2020

(C) The Author(s) 2020

\begin{abstract} types of analysis software on values and quantification of myocardial strain in healthy adults. segmental strain values were compared to each other regarding their post-processing approach and analysis software package. vs. $-17.1 \pm 1.8 \%, p=0.029$ and radial strain $30.7 \pm 4.7 \%$ vs. $27.8 \pm 4.6 \%, p=0.047$ ). be warranted.

Trial registration Retrospectively registered

Key Points

- Myocardial deformation assessed by feature tracking depends on the segmentation procedure.

- Global myocardial strain values differ significantly among vendors.

- Standardization in post-processing using CMR feature tracking is essential.
\end{abstract}

Objectives Quantification of myocardial deformation by feature tracking is of growing interest in cardiovascular magnetic resonance. It allows the assessment of regional myocardial function based on cine images. However, image acquisition, post-processing, and interpretation are not standardized. We aimed to assess the influence of segmentation procedure such as slice selection and different

Methods Healthy volunteers were retrospectively analyzed. Post-processing was performed using $\mathrm{CVI}^{42}$ and TomTec. Longitudinal and radial $_{\text {Long axis (LAX) }}$ strain were quantified using 4-chamber-view, 3-chamber-view, and 2-chamber-view. Circumferential and radial $_{\text {Short axis (SAX) }}$ strain were assessed in basal, midventricular, and apical short-axis views and using full coverage. Global and

Results We screened healthy volunteers studied at 1.5 or $3.0 \mathrm{~T}$ and included 67 (age $44.3 \pm 16.3$ years, 31 females). Circumferential and radial $\mathrm{SAX}_{\mathrm{X}}$ strain values were different between a full coverage approach vs. three short slices $(-17.6 \pm$ $1.8 \%$ vs. $-19.2 \pm 2.3 \%$ and $29.1 \pm 4.8 \%$ vs. $34.6 \pm 7.1 \%$ ). Different analysis software calculated significantly different strain values. Within the same vendor, different field strengths $(-17.0 \pm 2.1 \%$ at $1.5 \mathrm{~T}$ vs. $-17.0 \pm 1.7 \%$ at $3 \mathrm{~T}, p=0.845)$ did not influence the calculated global longitudinal strain (GLS), and were similar in gender $(-17.4 \pm 2.0 \%$ in females vs. $-16.6 \pm 1.8 \%$ in males, $p=0.098$ ). Circumferential and radial strain were different in females and males (circumferential strain $-18.2 \pm 1.7 \%$

Conclusions Myocardial deformation assessed by feature tracking depends on segmentation procedure and type of analysis software. Circumferential ${ }_{\mathrm{SAX}}$ and radial $\mathrm{SAX}_{\mathrm{X}}$ depend on the number of slices used for feature tracking analysis. As known from other imaging modalities, GLS seems to be the most stable parameter. During follow-up studies, standardized conditions should

Keywords Magnetic resonance imaging $\cdot$ Left ventricular function $\cdot$ Software $\cdot$ Myocard $\cdot$ Healthy volunteers

Carolin Lim and Edyta Blaszczyk contributed equally to this work.

Jeanette Schulz-Menger

Jeanette.schulz-menger@charite.de

1 Working Group on Cardiovascular Magnetic Resonance, Experimental and Clinical Research Center, a Joint Cooperation Between the Charité - Universitätsmedizin Berlin, Department of Internal Medicine and Cardiology and the Max-Delbrueck Center for Molecular Medicine, and HELIOS Klinikum Berlin Buch,

Department of Cardiology and Nephrology, Berlin, Germany
2 DZHK (German Center for Cardiovascular Research), Partner Site, Berlin, Germany

3 Berlin Ultrahigh Field Facility at the Max-Delbrueck Center for Molecular Medicine, Berlin, Germany

4 Department of Cardiology, Clinic Agatharied, Ludwig-Maximilians University München, Hausham, Germany 


$\begin{array}{ll}\text { Abbreviations } \\ \text { AHA } & \text { American Heart Association } \\ \text { CMR } & \text { Cardiovascular magnetic resonance } \\ \text { CS } & \text { Circumferential strain } \\ \text { GCS } & \text { Global circumferential strain } \\ \text { CV } & \text { Chamber view } \\ \text { GLS } & \text { Global longitudinal strain } \\ \text { GRE } & \text { Gradient echo sequence } \\ \text { LAX } & \text { Long axis } \\ \text { LGE } & \text { Late gadolinium enhancement } \\ \text { LV } & \text { Left ventricular } \\ \text { LVEDVI } & \text { Left ventricular end-diastolic index } \\ \text { LVEF } & \text { Left ventricular ejection fraction } \\ \text { LVOT } & \text { Left ventricular outflow tract } \\ \text { MOLLI } & \text { Modified look-locker inversion-recovery } \\ \text { RS } & \text { Radial strain } \\ \text { SAX } & \text { Short axis } \\ \text { SD } & \text { Standard deviation } \\ \text { SSFP } & \text { Steady-state free precession }\end{array}$

\section{Background}

Quantification of myocardial deformation applying myocardial strain is of growing interest in cardiovascular magnetic resonance (CMR). For a few years, it has been applied in research, and different vendors have developed postprocessing tools [1].

Myocardial strain allows quantitative measurement of global but also regional myocardial function and deformation offering additional information beyond ejection fraction [2, 3]. It enables early detection of subclinical myocardial dysfunction in patients with ischemic and non-ischemic heart disease and in preserved ejection fraction without wall motion abnormalities [2, 4-14].

Left ventricular deformation can be quantified in three dimensions: longitudinal and circumferential strain which show ventricular shortening in longitudinal and circumferential directions (negative strain) and radial strain that characterizes wall thickening (positive strain) [15].

Assessment of myocardial regional function is well known in echocardiography using speckle tracking $[12,15,16]$ but is also increasingly investigated in CMR using different techniques, such as strain encoding (SENC) [17, 18], displacement encoding (DENSE) [19], and tagging [17, 18, 20-22]. Feature tracking is a tool which in contrast to the methods mentioned above enables post-processing analysis of myocardial strain based on routine steady-state free precession (SSFP) cine images as acquired for the assessment of left ventricular (LV) function and volume [8, 16, 23]. It avoids acquisition of additional images and saves time [23]. Preexisting contours for calculation of LV function can be used for strain analysis making it a timesaving method. For those reasons, feature tracking seems to be a beneficial tool, e.g., for follow-up examinations.

Even though publications regarding CMR strain analysis exist, standards for image acquisition and interpretation are still not established. Different vendors and different analysis procedures such as slice selection procedures, even within the same software, can heavily influence deformation values. This may lead to uncertainties in comparison and interpretation of data. We aimed to analyze the influence of segmentation procedure such as slice selection on values of quantification of myocardial strain in healthy adults. Additionally, we intended to analyze the influence of different software packages and to provide regional strain quantification.

\section{Methods}

\section{Study population}

We retrospectively screened 243 truly healthy subjects, who were prospectively examined in former studies [24-28]. Exclusion criteria were known cardiovascular risk factors, any pre-existing diseases or medications, impaired LV ejection fraction (LVEF) $(<55 \%)$, or pathological findings in 12 lead ECG or CMR. Incomplete CMR data for feature tracking analysis led to exclusion. That included lack of long-axis (LAX) or short-axis (SAX) slices $(n=137)$ or variable number of cardiac phases $(n=41)$. The ethics committee approved all studies. Informed written consent was obtained in concordance with the Helsinki Declaration.

\section{CMR acquisition}

CMR was performed at 1.5-T and 3-T scanners. At $1.5 \mathrm{~T}$ (Magnetom Avanto), a 12-channel radio frequency coil was used and at $3 \mathrm{~T}$ (Magnetom Verio, both Siemens Healthineers) a 32-channel radio frequency coil. SSFP cine images were acquired during repeated breath-holds for LV in 4-chamberview (4CV), 3-chamber-view (3CV), 2-chamber-view (2CV), and at least three SAX slices (SAX full coverage and/or three SAX slices in basal, midventricular, and apical plane). Recently, detailed sequence parameters were published [24-29]: at $1.5 \mathrm{~T}$ : repetition time $2.8 \mathrm{~ms}$, slice thickness $6 \mathrm{~mm}$, flip angle 80 degrees, echo time $1.2 \mathrm{~ms}$, field of view $276 \times 340 \mathrm{~mm}^{2}$, matrix $156 \times 192$, voxel size $1.4 \times 1.4 \times$ $7 \mathrm{~mm}, 30$ cardiac phases; and at $3 \mathrm{~T}$ : repetition time $3.1 \mathrm{~ms}$, slice thickness $6 \mathrm{~mm}$, flip angle 45 degrees, echo time $1.3 \mathrm{~ms}$, field of view $276 \times 340 \mathrm{~mm}^{2}$, matrix $156 \times 192$, voxel size $1.4 \times 1.4 \times 7 \mathrm{~mm}, 30$ cardiac phases.

Two independent experienced readers (SCMR level III) performed the visual evaluation of the cine images. 
LV function and volumes were quantified in a whole $S A X$ stack according to the recommendation of the SCMR [30] applying $\mathrm{CVI}^{42}$ software (Version 4.1.2, Circle Cardiovascular Imaging Inc.). Endo- and epicardial contours were manually drawn in end-diastolic and end-systolic phase. Papillary muscles were excluded from the LV volume.

\section{Feature tracking}

Feature tracking analysis was performed retrospectively using $\mathrm{CVI}^{42}$ software (prototype version 5.3.0, Circle Cardiovascular Imaging Inc.). Longitudinal strain and radial $_{\text {LAX }}$ strain (RS) were assessed in three LAX views: $4 \mathrm{CV}, 3 \mathrm{CV}$, and $2 \mathrm{CV}$ (Fig. 1). Circumferential strain (CS) and $\mathrm{RS}_{\mathrm{SAX}}$ were analyzed using three SAX slices (basal, midventricular, and apical) in all subjects (Fig. 1). If available, strain was additionally assessed using a SAX full coverage (Fig. 2). Endo- and epicardial contours were manually drawn in end-diastolic phase, defined as the phase with the largest LV volume. End-diastolic phase had to be identical in all SAX and LAX slices of one subject. Trabeculae, papillary muscles, pericardium, and epicardial fat were consequently excluded from contouring. Left ventricular outflow tract (LVOT) was completely excluded in all SAX slices if seen in diastolic and/ or systolic phases (Fig. 2). 2D strain analysis was assessed globally and segmentally for longitudinal, $\mathrm{RS}_{\mathrm{LAX}}$, CS, and $\mathrm{RS}_{\mathrm{SAX}}$ strain. Segmentation included both possibilities of slice selection (three slices versus the whole stack) and the segmentation of the left ventricle according to the AHA 17segment model [31]. We excluded the apex (segment 17) from feature tracking analysis; so far, the 16 segment model was used. Tracking quality and segmentation were evaluated using software tools like mesh, boundaries, or myocardial points. If contours did not follow the epi- or endocardial borders correctly, delineation was retraced and adjusted. In case of remaining tracking issues, all corresponding segments were excluded. Also, incorrect segmentation (see Fig. 3) led to exclusion. Excluded segments were not considered for global strain assessment.

Strain results were compared between field strengths $(1.5 \mathrm{~T}$ and $3 \mathrm{~T}$ ) and between different numbers of SAX slices (three SAX slices versus full coverage) in CS and $\mathrm{RS}_{\mathrm{SAX}}$, as well as RS between LAX and SAX analysis.

Bulls-eye plots visualizing segmental strain values were created using the Python package Matplotlib.

Global strain analysis was repeated by the same observer (intra-observer) and by a different observer (inter-observer) in the same randomly selected subjects $(n=10)$.

\section{Software comparison}

All images were also analyzed with TomTec Image Arena (version 1.3.0.91, TomTec Imaging Systems $\mathrm{GmbH}$ ) (Fig. 4). 4CV, $3 \mathrm{CV}$, and $2 \mathrm{CV}$ were used for longitudinal and transversal (radial ${ }_{\text {LAX }}$ ) strain. CS and $\mathrm{RS}_{\mathrm{SAX}}$ were assessed using three SAX slices (basal, midventricular, and apical). Endo- and epicardial contours were manually drawn in end-diastolic and endsystolic phases. Trabeculae and papillary muscles were excluded from analysis, as well as LVOT. Tracking quality was checked manually, specifically whether contours followed endo- and epicardial borders correctly and were adjusted if necessary. Myocardial strain was analyzed on a global and segmental level.
Fig. 1 Post-processing using 2D strain analysis by $\mathrm{CVI}^{42}$. Endo(red) and epicardial (green) contours were manually drawn in end-diastolic phase in long axis $(\mathbf{a}-\mathbf{c})$ and short axis $(\mathbf{d}-\mathbf{f})$. 4chamber-view (a), 3-chamberview (b), and 2-chamber-view (c) were included in long-axis strain analysis. For short-axis strain, contours were drawn in three short-axis slices: basal (d), midventricular (e), and apical (f)
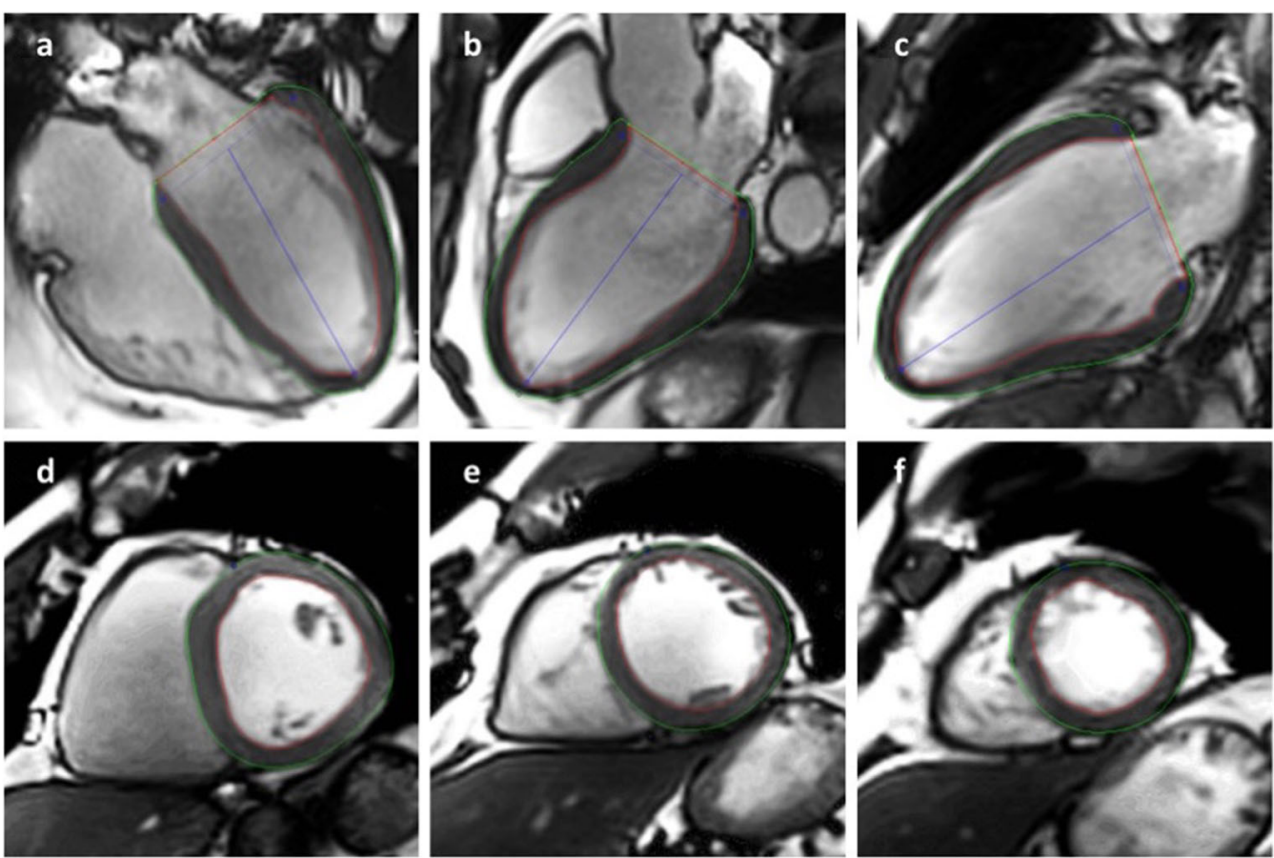

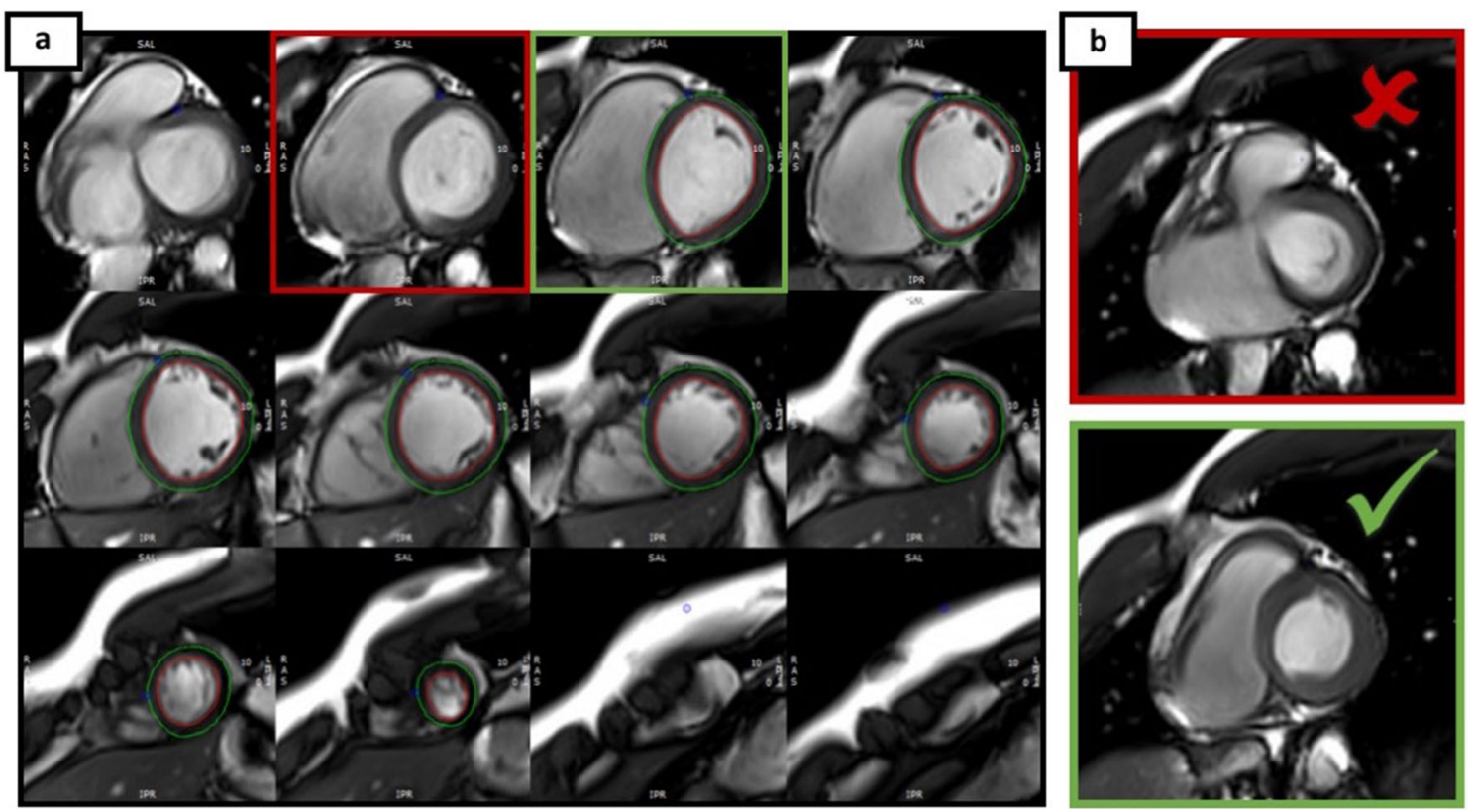

Fig. 2 Strain analysis using full coverage $\left(\mathrm{CVI}^{42}\right)$. Endo- and epicardial contours were drawn in end-diastolic phase (a). If LVOT was visible in end-systolic phase (b, marked red), slices were excluded. The first slice

Three LAX (4CV, 3CV, 2CV) and three SAX slices using the exact same slice number were considered for software comparison.

\section{Statistical analysis}

Statistical analyses were performed using IBM SPSS Statistic version 23 . We calculated mean values and standard deviation (SD) as well as median and interquartile ranges (IQR) for demographic parameters, LV function, and strain measurements. Volumes were indexed to body surface area (BSA) and height. The non-parametric Mann-Whitney $U$ test for unpaired samples was used for comparisons of strain parameters between gender, analysis software, and field strength. Differences were considered to be statistically significant at $p<0.05$. Intra- and inter-observer reproducibility were used for analysis was chosen as the most basal slice that did not show LVOT in any end-diastolic (a, marked green) and end-systolic phase (b, marked green)

analyzed using intra-class correlation coefficient (ICC) and $95 \%$ confidence interval (CI). ICC was classified as poor (ICC $<0.4)$, good $(\mathrm{ICC}=0.4-0.75)$, or excellent $(\mathrm{ICC}$ $>0.75)[1]$.

\section{Results}

\section{Basic data}

Sixty-seven healthy subjects ( $n=36$ at $1.5 \mathrm{~T}$ and $n=31$ at $3 \mathrm{~T}$ ) were included and analyzed (mean age $44.3 \pm 16.3$ years, $n=$ 31 females). The proportion of men and age between the field strength groups was equalized. The $1.5 \mathrm{~T}$ group had 19 (52.8\%), while the $3 \mathrm{~T}$ group accounted for 17 (54.8\%) male
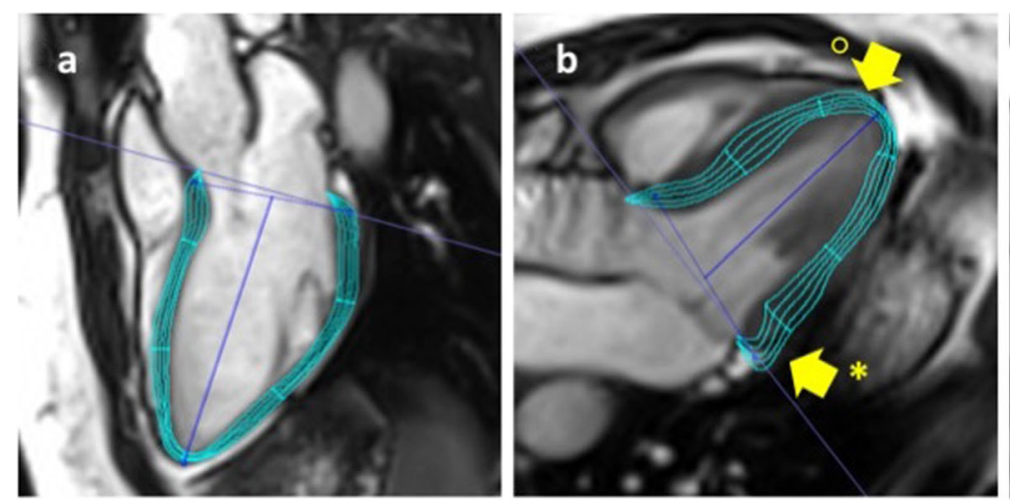

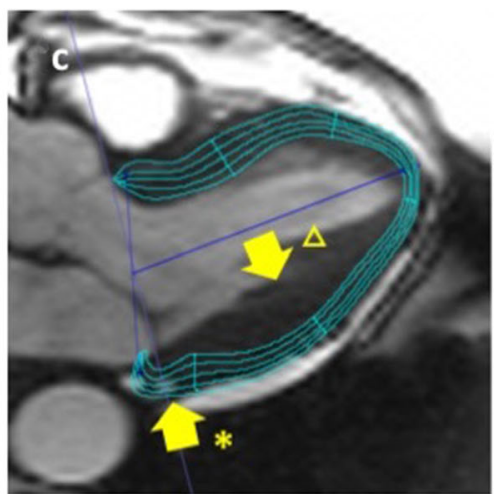

Fig. 3 Quality assessment for accurate tracking and correct segmentation applying $\mathrm{CVI}^{42}$. a Optimal segmentation. b and $\mathbf{c}$ show incorrect segmentations in 3-chamber-view: the basal inferolateral segments are relatively short $(*)$ and the apical septal segment extends to apical lateral $\left({ }^{\circ}\right)$. Additionally, contours do not follow endocardial borders accurately $(\Delta)$ 
Fig. 4 2D strain analysis of the left ventricle using different postprocessing software. Strain was analyzed using $\mathrm{CVI}^{42}(\mathbf{a}-\mathbf{b})$ and TomTec software $(\mathbf{c}-\mathbf{d})$.

Longitudinal and radial $\mathrm{LAX}_{\mathrm{LAX}}$ strain were assessed in $4 \mathrm{CV}, 3 \mathrm{CV}(\mathbf{a}, \mathbf{c})$ and $2 \mathrm{CV}$; circumferential and radial $_{\mathrm{SAX}}$ strain were analyzed in basal (b, d), medial, and apical short-axis slice
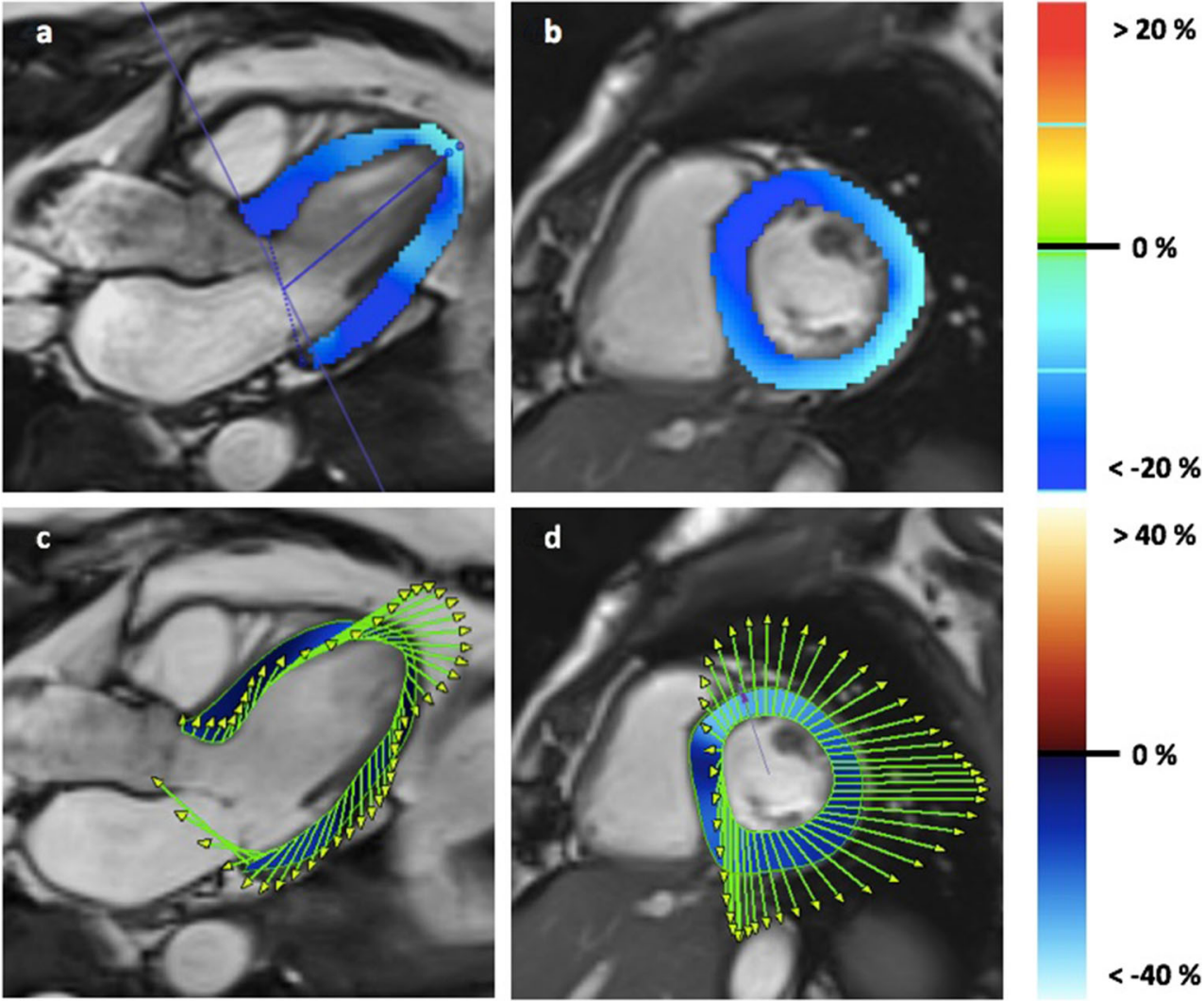

$>40 \%$

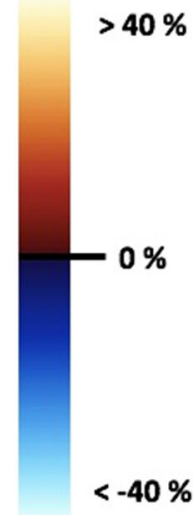

Table 1

Basic characteristics of the study population

subjects. Mean age was $45.0 \pm 16.39$ years at $1.5 \mathrm{~T}$ versus $43.48 \pm 16.33$ years at $3 \mathrm{~T}(p=0.739)$.

All volunteers had normal LV function (LVEF 64.1 \pm $4.2 \%$ ) without wall motion abnormalities. Demographic parameters as well as LV function and volumes are summarized in Table 1. Seven subjects had to be excluded from 3D LV function analysis due to incomplete SAX package $(n=6)$ or artifacts $(n=1)$.

\section{Feature tracking quality}

In all 67 subjects, strain was analyzed in $4 \mathrm{CV}, 3 \mathrm{CV}, 2 \mathrm{CV}$, and three SAX slices. Sixty-one subjects were additionally analyzed by $\mathrm{CVI}^{42}$ using a full coverage. Using $\mathrm{CVI}^{42}$, we could include 1020 segments $(95.1 \%)$ for longitudinal strain and 1033 segments $(96.4 \%)$ for $\mathrm{RS}_{\mathrm{LAX}}$. In total, 1064 segments (99.3\%) for $\mathrm{RS}_{\mathrm{SAX}}$ and 1064 segments $(99.3 \%)$ for CS were analyzed. In the SAX, strain analysis using SAX full coverage 966 segments $(99.0 \%)$ from each of $\mathrm{RS}_{\mathrm{SAX}}$ and CS could be included.

For analysis with TomTec, 1059 segments $(98.8 \%)$ could be included for longitudinal strain, 1056 segments $(98.5 \%)$ for $\mathrm{RS}_{\mathrm{LAX}}, 1071$ segments $(99.9 \%)$ for $\mathrm{RS}_{\mathrm{SAX}}$, and $1070 \mathrm{seg}-$ ments $(99.8 \%)$ for CS.

Reasons for exclusion were inaccurate tracking or incorrect segmentation.

\begin{tabular}{lcrrr}
\hline & Mean \pm SD & Median & Q1 & Q3 \\
\hline Demographic parameters & & & & \\
Gender (female $\mid$ male) & $31 \mid 36$ & & & \\
Age $($ years $)$ & $44.3 \pm 16.3$ & 45.0 & 28.0 & 59.0 \\
Height $(\mathrm{cm})$ & $174.1 \pm 8.6$ & 173.0 & 168.0 & 180.0 \\
Weight $(\mathrm{kg})$ & $74.4 \pm 13.0$ & 73.0 & 64.0 & 81.6 \\
BMI $\left(\mathrm{kg} / \mathrm{m}^{2}\right)$ & $24.6 \pm 3.9$ & 24.2 & 21.8 & 27.1 \\
BSA $\left(\mathrm{m}^{2}\right)$ & $1.9 \pm 0.2$ & 1.9 & 1.8 & 2.0 \\
HR $(1 / \mathrm{min})$ & $72.8 \pm 11.7$ & 71.0 & 65.8 & 79.1 \\
Systolic BP (mmHg) & $128.1 \pm 14.1$ & 128.0 & 117.0 & 137.0 \\
Diastolic BP $(\mathrm{mmHg})$ & $73.6 \pm 11.7$ & 75.0 & 68.0 & 79.0 \\
LV function and volumes & & & & \\
LVEF $(\%)$ & $64.1 \pm 4.2$ & 64.0 & 60.4 & 67.2 \\
LVEDV $(\mathrm{ml})$ & $139.9 \pm 33.2$ & 135.0 & 112.2 & 157.9 \\
LVEDVI (ml/m $\left.{ }^{2}\right)$ & $74.5 \pm 15.1$ & 76.1 & 62.6 & 83.3 \\
LVEDVI (ml/cm) & $0.8 \pm 0.2$ & 0.8 & 0.7 & 0.9 \\
LVESV $(\mathrm{ml})$ & $51.1 \pm 15.0$ & 47.5 & 40.8 & 60.4 \\
LVM $(\mathrm{g})$ & $101.0 \pm 22.3$ & 97.9 & 88.7 & 110.9 \\
LVMI $\left(\mathrm{g} / \mathrm{m}^{2}\right)$ & $53.7 \pm 9.0$ & 54.2 & 48.3 & 57.7 \\
LVMI $(\mathrm{g} / \mathrm{cm})$ & $0.6 \pm 0.1$ & 0.6 & 0.5 & 0.6 \\
SV $(\mathrm{ml})$ & $89.2 \pm 20.2$ & 88.0 & 75.6 & 101.1 \\
SVI $\left(\mathrm{ml} / \mathrm{m}^{2}\right)$ & $47.5 \pm 9.4$ & 46.6 & 39.2 & 54.8 \\
SVI $(\mathrm{ml} / \mathrm{cm})$ & $0.5 \pm 0.1$ & 0.5 & 0.4 & 0.6 \\
\hline
\end{tabular}

Data are shown as mean values \pm standard deviation (SD), median, and interquartile ranges $(\mathrm{Q} 1$ and $\mathrm{Q} 3)$

$B M I$ body mass index, $B S A$ body surface area (Mosteller), $H R$ heart rate, $B P$ blood pressure, $L V E F$ left ventricular ejection fraction, $L V E D V$ left ventricular end-diastolic volume, $L V E D V I$ left ventricular end-diastolic volume index, $L V E S V$ left ventricular end-systolic volume, $L V M$ left ventricular mass, $L V M I$ left ventricular mass index, $S V$ stroke volume, $S V I$ stroke volume index 


\section{Influence of slice selection on circumferential and radial $_{\mathrm{SAX}}$ strain using $\mathrm{CVI}^{42}$}

$\mathrm{CS}$ and $\mathrm{RS}_{\mathrm{SAX}}$ measurements assessed by a stack of short axes covering the whole LV differ significantly from those assessed using three short axes: global CS $-19.2 \pm 2.3 \%$ (median $-19.0 \%$, IQR -20.6 to $-17.9 \%$ ) in 3 SAX vs. $-17.6 \pm$ $1.8 \%$ (median $-17.7 \%$, IQR -18.6 to $-16.7 \%$ ) in full coverage $(p<0.001)$ and global $\mathrm{RS}_{\mathrm{SAX}} 34.6 \pm 7.1 \%$ (median $33.4 \%$, IQR $29.9-38.8 \%$ ) in 3 SAX vs. $29.1 \pm 4.8 \%$ (median $29.1 \%$, IQR $26.2-31.9 \%$ ) in full coverage $(p<0.001)$ (for details, see Table 2).

Using three SAX slices, no differences were found for global strain measurements between $1.5 \mathrm{~T}$ and $3 \mathrm{~T}$ : global CS $-19.6 \pm 2.3 \%$ (median $-18.9 \%$, IQR -21.3 to $-18.0 \%$ ) at $1.5 \mathrm{~T}$ vs. $-18.8 \pm 2.2 \%$ (median $-19.0 \%$, IQR -20.5 to $-17.6 \%)$ at $3 \mathrm{~T}(p=0.263)$ and for global $\mathrm{RS}_{\mathrm{SAX}} 36.0 \pm 7.5 \%$ (median $33.4 \%$, IQR $29.9-38.8 \%$ ) at $1.5 \mathrm{~T}$ vs. $33.0 \pm 6.2 \%$ (median 32.6\%, IQR 28.9-37.3\%) at $3 \mathrm{~T}(p=0.128)$. However, some segmental strain values differed significantly between field strengths for both CS and $\mathrm{RS}_{\mathrm{SAX}}$ (for details, see supplemental material online additional file 1).

Using SAX full coverage, both global and segmental strain values did not show any significant difference between field strengths: CS $-17.7 \pm 1.8 \%$ (median $-17.7 \%$, IQR -18.8 to $-16.6 \%$ ) at $1.5 \mathrm{~T}$ vs $-17.6 \pm 1.8 \%$ (median $-17.6 \%$, IQR -18.5 to $-16.9 \%)$ at $3 \mathrm{~T}(p=0.85)$ and global $\mathrm{RS}_{\mathrm{SAX}} 29.4$ $\pm 5.1 \%$ (median $29.6 \%$, IQR $25.9-32.3 \%$ ) at $1.5 \mathrm{~T}$ vs $28.7 \pm$ $4.5 \%$ (median $28.7 \%$, IQR $26.6-31.1 \%)$ at $3 \mathrm{~T}(p=0.665)$ (for details, see supplemental material online additional file 1).

In both, three selected slices and a whole SAX stack global circumferential and radial $_{S A X}$ strain differed significantly between genders (for details, see Table 2). Gender-related strain values are visualized in the supplemental material additional file 2 .

\section{Assessment of radial strain in long- and short-axis views}

Global radial strain acquired in LAX ( radial $_{\text {LAX }}$ ) versus SAX

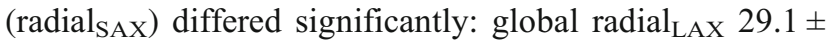
$5.3 \%$ (median $29.1 \%$, IQR 25.1-32.8\%) versus global radial $_{\mathrm{SAX}} 34.6 \pm 7.1 \%$ (median $33.4 \%$, IQR $29.9-38.8 \%$ ) $(p<0.001)$.

\section{Longitudinal strain using $\mathrm{CVI}^{42}$}

Longitudinal strain did not show any significant difference for both global and segmental strain measurements between $1.5 \mathrm{~T}$ and $3 \mathrm{~T}$ : $-17.0 \pm 2.1 \%$ (median $-17.0 \%$, IQR -18.4 to $-15.3 \%$ ) vs. $-17.0 \pm 1.7 \%$ (median $-17.1 \%$, IQR -18.0 to $-15.8 \%$ ) ( $p=0.845$ accordingly). No significant differences have been found between females and males: $-17.4 \pm 2.0 \%$ (median $-17.7 \%$, IQR -18.4 to $-15.8 \%$ ) and $-16.6 \pm 1.8 \%$ (median $-16.6 \%$, IQR -18.0 to $-15.3 \%)(p=0.098)$. On a segmental level, only AHA segment 5 (basal inferolateral) showed a significant difference between genders: $-25.8 \pm$ $5.9 \%$ (median $-26.7 \%$, IQR -30 to $-22.8 \%$ ) in females versus $-23.3 \pm 5.0 \%$ (median $-23.4 \%$, IQR -27.5 to $-18.4 \%)$ in males $(p=0.048)$. Segmental strain measurements for longitudinal strain are presented in Fig. 5.

\section{Software comparison}

Strain measurements assessed with TomTec software were significantly different to those assessed with $\mathrm{CVI}^{42}$ (Table 3). GLS was $-17.0 \pm 1.9 \%$ (median $-17.0 \%$, IQR -18.4 to $-15.6 \%$ ) for $\mathrm{CVI}^{42}$ and $-20.5 \pm 2.7 \%$ (median $-20.2 \%$, IQR -22.6 to $-18.8 \%)$ for TomTec $(p<0.001)$. Significant differences were also found for most segmental strain values (for details, see supplemental material online additional files 3-6).

Gender-related global strain values using TomTec are summarized in Table 2. Unlike differences in global $\mathrm{RS}_{\mathrm{SAX}}$, GLS and global CS were not associated with gender.

\section{Intra- and inter-observer reproducibility $\left(\mathrm{CV}^{\mathbf{4 2}}\right)$}

GLS reproducibility was as follows: ICC was $0.941(95 \% \mathrm{CI}$ 0.759-0.985) for intra-observer and 0.829 (95\% CI 0.273 0.958 ) for inter-observer analysis. We observed an excellent intra- and inter-observer reproducibility across all global strain measurements (for details, see supplemental material 7). Intraobserver agreement was best for CS (ICC 0.977, 95\% CI $0.907-0.994$ ) and lowest for $\mathrm{RS}_{\mathrm{LAX}}$ (ICC $0.930,95 \% \mathrm{CI}$ 0.715-0.983). Inter-observer agreement was best for radial $_{\mathrm{SAX}}$ strain (ICC $0.975,95 \%$ CI $0.889-0.994$ ) and lowest for longitudinal strain (ICC 0.829, 95\% CI 0.273-0.958).

\section{Discussion}

In this study, we aimed to increase knowledge about influencing factors on strain results obtained by CMR feature tracking. We focused on the segmentation procedure and on the comparison of software packages of two different vendors.

For the first time, we showed that CS and $\mathrm{RS}_{\mathrm{SAX}}$ were dependent on the number of slices used for feature tracking analysis. Previous published studies considered a different number of slices for strain analysis making it difficult to compare strain values to each other. While some used one LAX and one midventricular SAX slice [20,32,33], others included two LAX and three SAX views [34, 35] or considered all three LAX views and a SAX full coverage [36]. The variation in analysis procedure like slice selection may lead to different quantitative results and consequently to uncertainties and 


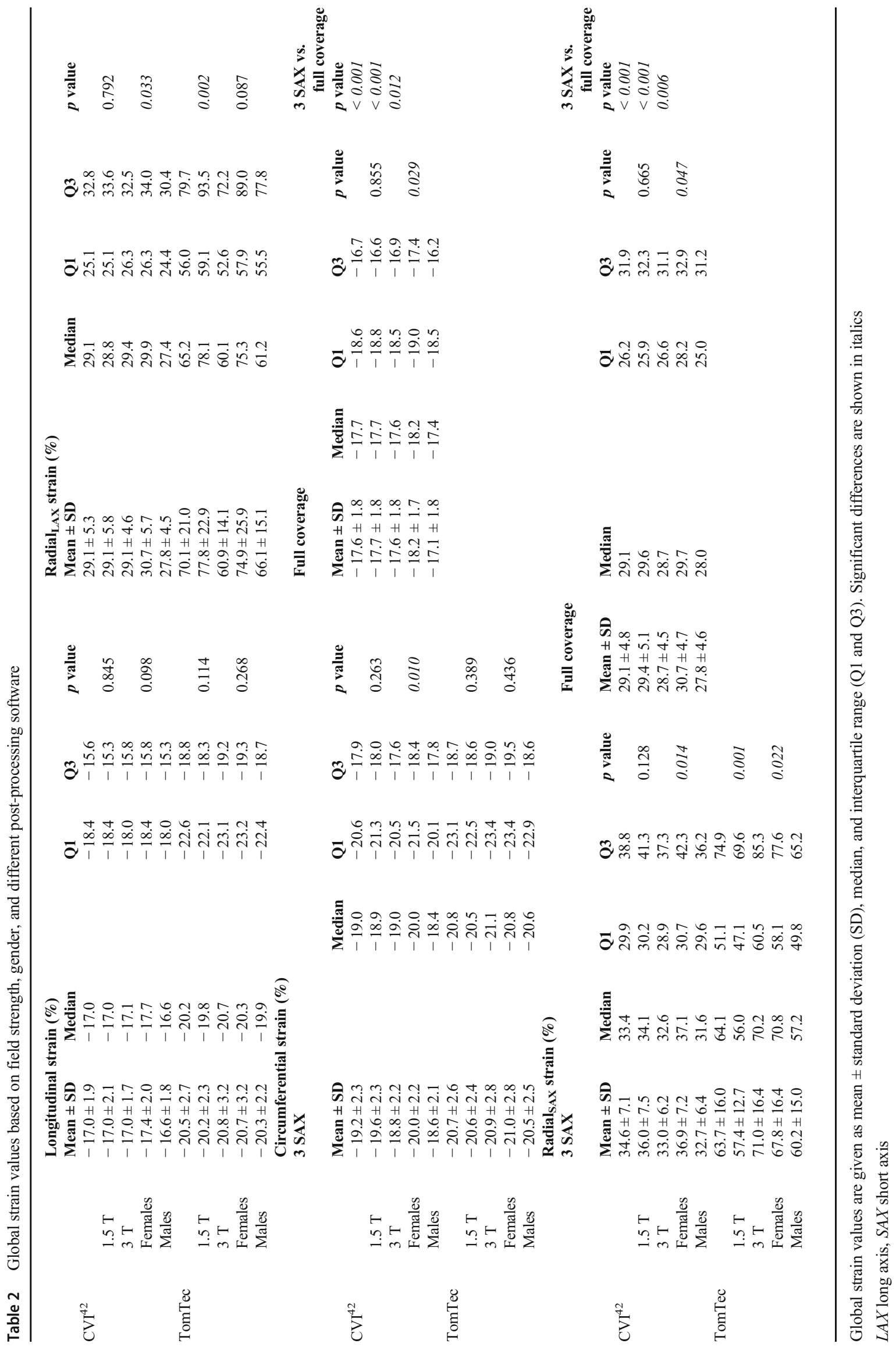



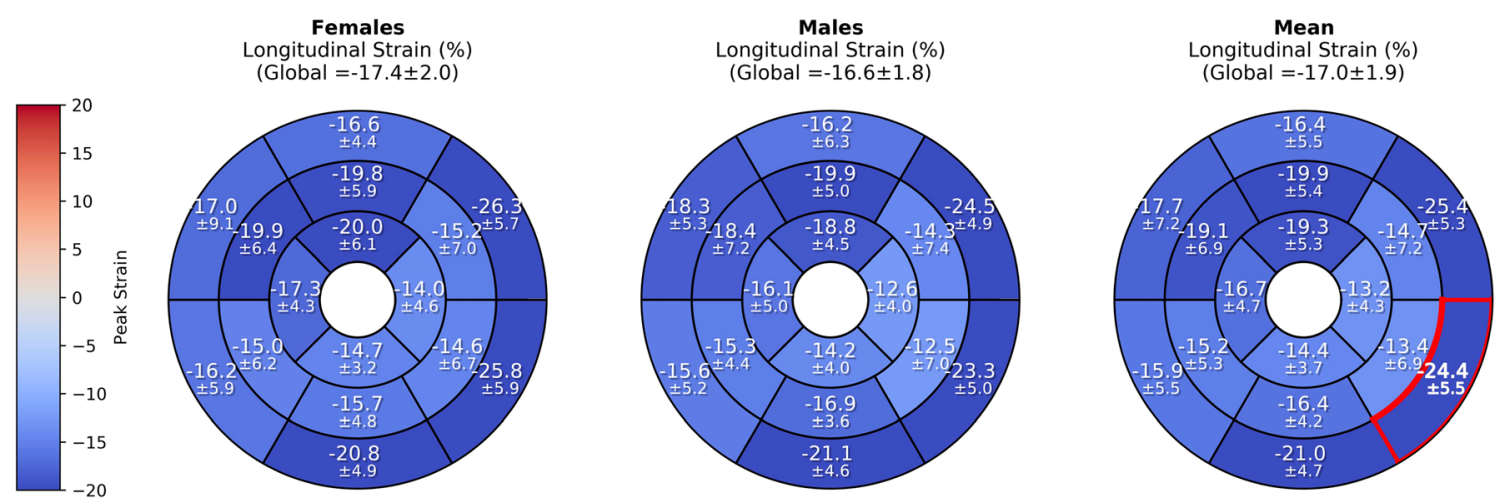

Fig. 5 Gender-related mean values for longitudinal strain using $\mathrm{CVI}^{42}$. Segmental values are provided as mean (in \%) \pm standard deviation in a bulls-eye plot according to the AHA segment model [31]. Segment 5 (marked red) differed between genders $(p=0.048)$

difficulties in comparison and interpretation. Significant variations among vendors are already known in echocardiography and CMR-FT and this should be considered when performing serial studies [37]. A recent study by Liu et al compared 3D strain analysis (three LAX slices and SAX full coverage) with 2D analysis using one horizontal LAX and one midventricular SAX slice showing notable differences [38]. In our study, we detected differences for CS and $\mathrm{RS}_{\mathrm{SAX}}$ between three SAX slices and full coverage using $\mathrm{CVI}^{42}$. Of note, both parameters were significantly higher using $3 \mathrm{SAX}$ slices vs. full coverage; one should assume that partial volume effects, mainly effecting an apical slice, may influence the results. Furthermore, vendors may use a different way of pixel definition leading to a different boundary detection.

Radial strain assessed in LAX and SAX slices differed significantly. There is no broad experience in using radial $_{\text {LAX }}$ strain yet, but when SAX slices are missing, assessment of radial strain in LAX can add information.

Among different types of post-processing software, both global and segmental strain values differed significantly.
These findings indicate that strain values are not comparable between different software applications. Our findings in terms of differences among post-processing software packages are mostly in accordance with previous published data $[1,20,38]$. Barreiro-Pérez et al showed variability among different vendors (TomTec, $\mathrm{CVI}^{42}$, Medis, Medviso) in GLS and RS measurements, but not in CS [1]. In our study, strain values were significantly lower using $\mathrm{CVI}^{42}$, but these findings conform with previous studies [20,38]. Cao et al compared different sequences and different post-processing software [20], detecting notable differences between all CMR techniques. However, the proper validation of most analysis procedures as well as absolute and objective reference values is yet to be established. While DENSE, SENC, and tagging, techniques for measuring three-dimensional motion and deformation, require dedicated sequences, feature tracking analysis is based on routine SSFP cine images. However, FT is based on contours only and does not follow intrinsic myocardial contraction. Moreover, the influence of field strengths seems to not be relevant. Schuster et al showed similar results for myocardial

Table 3 Global myocardial strain compared between different post-processing software

\begin{tabular}{|c|c|c|c|c|c|c|c|c|c|c|}
\hline & & \multicolumn{4}{|l|}{$\mathrm{CVI}^{42}$} & \multicolumn{4}{|l|}{ TomTec } & \multirow[t]{2}{*}{$p$ value } \\
\hline & & Mean \pm SD & Median & Q1 & Q3 & Mean \pm SD & Median & Q1 & Q3 & \\
\hline \multirow[t]{3}{*}{ Global longitudinal strain (\%) } & & $-17.0 \pm 1.9$ & $-17.0 \pm 1.9$ & -17.0 & -18.4 & $-20.5 \pm 2.7$ & -20.2 & -22.6 & -18.8 & $<0.001$ \\
\hline & $1.5 \mathrm{~T}$ & $-17.0 \pm 2.1$ & $-17.0 \pm 2.1$ & -17.0 & -18.4 & $-20.2 \pm 2.3$ & -19.8 & -22.1 & -18.3 & $<0.001$ \\
\hline & $3 \mathrm{~T}$ & $-17.0 \pm 1.7$ & $-17.0 \pm 1.7$ & -17.1 & -18.0 & $-20.8 \pm 3.2$ & -20.7 & -23.1 & -19.2 & $<0.001$ \\
\hline \multirow[t]{3}{*}{ Global circumferential strain (\%) } & & $-19.2 \pm 2.3$ & -19.0 & -20.6 & -17.9 & $-20.7 \pm 2.6$ & -20.8 & -23.1 & -18.7 & 0.001 \\
\hline & $1.5 \mathrm{~T}$ & $-19.6 \pm 2.3$ & -18.9 & -21.3 & -18.0 & $-20.6 \pm 2.4$ & -20.5 & -22.5 & -18.6 & 0.076 \\
\hline & $3 \mathrm{~T}$ & $-18.8 \pm 2.2$ & -19.0 & -20.5 & -17.6 & $-20.9 \pm 2.8$ & -21.1 & -23.4 & -19.0 & 0.001 \\
\hline \multirow[t]{3}{*}{ Global radial $_{\mathrm{SAX}}$ strain $(\%)$} & & $34.6 \pm 7.1$ & 33.4 & 29.9 & 38.8 & $63.7 \pm 16.0$ & 64.1 & 51.1 & 74.9 & $<0.001$ \\
\hline & $1.5 \mathrm{~T}$ & $36.0 \pm 7.5$ & 34.1 & 30.2 & 41.3 & $57.4 \pm 12.7^{*}$ & 56.0 & 47.1 & 69.6 & $<0.001$ \\
\hline & $3 \mathrm{~T}$ & $33.0 \pm 6.2$ & 32.6 & 28.9 & 37.3 & $71.0 \pm 16.4^{*}$ & 70.2 & 60.5 & 85.3 & $<0.001$ \\
\hline \multirow[t]{3}{*}{ Global radial $_{\text {LAX }}$ strain $(\%)$} & & $29.1 \pm 5.3$ & 29.1 & 25.1 & 32.8 & $70.1 \pm 21.0$ & 65.2 & 56.0 & 79.7 & $<0.001$ \\
\hline & $1.5 \mathrm{~T}$ & $29.1 \pm 5.8$ & 28.8 & 25.1 & 33.6 & $77.8 \pm 22.9^{*}$ & 78.1 & 59.1 & 93.5 & $<0.001$ \\
\hline & $3 \mathrm{~T}$ & $29.1 \pm 4.6$ & 29.4 & 26.3 & 32.5 & $60.9 \pm 14.1^{*}$ & 60.1 & 52.6 & 72.2 & $<0.001$ \\
\hline
\end{tabular}

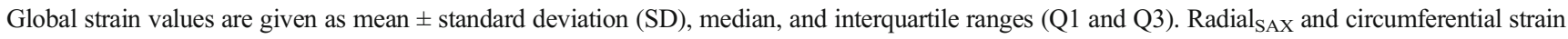
were assessed using three short-axis slices (basal, midventricular, apical). Significant differences $(p<0.05)$ are shown in italics. * $p<0.05$ between $1.5 \mathrm{~T}$ and $3 \mathrm{~T}$ within one software 
strain among $1.5 \mathrm{~T}$ and $3 \mathrm{~T}$ applying TomTec [32]. This agrees with our results since field strength did not influence global values of longitudinal, $\mathrm{RS}_{\mathrm{LAX}}, \mathrm{RS}_{\mathrm{SAX}}$, and CS strain using $\mathrm{CVI}^{42}$.

Reference values for CMR feature tracking analysis have been published, mainly focused on global left ventricular strain. Most studies performed feature tracking via TomTec $[36,39,40]$. Liu et al were the first to establish normal ranges for $\mathrm{CVI}^{42}$ using 3D strain analysis [38]. However, regional deformation was only acquired for CS. Regional assessment of myocardial strain is less validated, but may reveal further information compared to global values as single regions of the myocardium can be injured even though global strain is in normal range. We added knowledge on reference values for myocardial strain in healthy subjects using $\mathrm{CVI}^{42}$ and TomTec.

Unlike most studies showing greater deformation in females resulting in more negative strain [36, 39-42], we did not find gender-related differences for global longitudinal strain. The larger magnitudes of global CS in females having more negative strain values also agree with the findings reported by Andre et al and Peng et al [40, 41]. However, the higher global radial strain values in females contradict former findings $[36,40]$.

In accordance with our findings, CMR feature tracking has shown fair reproducibility in previous studies [34]. In fact, strain assessment is influenced by observer experience, but reproducibility may be optimized by training [43, 44]. Most studies indicate better reproducibility for global rather than segmental strain analysis with global CS being the most and global radial strain being the least reproducible measurement [20, 33, 35, 36, 42].

However, analysis methods throughout all studies were not standardized until now. CMR feature tracking-derived strain seems to be influenced by many factors including software package and the applied approach of image processing; thus, reference values should be derived from similar approaches. Currently, no gold standard exists. There is no defined "right" or "wrong" as in most of the publications that evaluate differences between post-processing software or sequences. But there is a need to understand that the application of different approaches may lead to different results.

CMR feature tracking is a promising tool that enables early detection of subtle myocardial dysfunction and prediction of major adverse cardiovascular events [5-7]. Standardization is needed if assessment of myocardial deformation including feature tracking should enter clinical routine.

\section{Limitations}

This study is limited by a relatively small, but carefully and well-characterized healthy study cohort. As our analysis was performed retrospectively in prospectively enrolled volunteers, scan protocols were slightly different. This led to exclusion of 176 subjects due to incomplete CMR data. This may be preventable by a prospectively designed study, but our settings also reflect potential difficulties in clinical routine.

Our statistical analysis was only descriptive and exploratory. It indicates that differences among vendors or segmentation procedures may exist, but further validation remains necessary.

The CMR examinations performed at $1.5 \mathrm{~T}$ and $3 \mathrm{~T}$ did not contain the same subjects, but showed an equal distribution regarding gender and age. In accordance with our results, preexisting studies have also shown that field strength does not influence global strain values [32].

CMR feature tracking is less validated for regional strain and radial $_{\text {LAX }}$ strain, but they can presumably reveal different physiological mechanisms of the myocardium. Regional assessment is limited by inaccurate tracking or incorrect segmentation which may distort segmental strain values. We provide numbers, but long-term studies have to show the potential significance before CMR-FT may enter clinical routine.

\section{Conclusion}

Myocardial deformation assessed by feature tracking depends on segmentation procedure and type of analysis software.

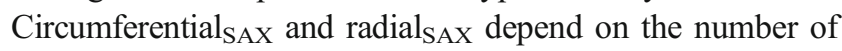
slices used for feature tracking analysis. As known from other imaging modalities, GLS seems to be the most stable parameter. Standardized conditions should be considered.

Supplementary Information The online version contains supplementary material available at https://doi.org/10.1007/s00330-020-07539-5.

Funding Open Access funding enabled and organized by Projekt DEAL.

\section{Compliance with ethical standards}

Guarantor The scientific guarantor of this publication is Prof. Jeanette Schulz-Menger.

Conflict of interest The authors of this manuscript declare no relationships with any companies whose products or services may be related to the subject matter of the article.

Statistics and biometry No complex statistical methods were necessary for this paper.

Informed consent Written informed consent was not required for this study because we screened healthy subjects, who were prospectively examined in former studies and written informed consent was obtained from all subjects (patients) in all former studies.

Ethical approval Institutional Review Board approval was not required because we screened healthy subjects, who were prospectively examined in former studies. The ethical committee had approved all former studies. 


\section{Methodology}

- retrospective

- observational

- performed at one institution

Open Access This article is licensed under a Creative Commons Attribution 4.0 International License, which permits use, sharing, adaptation, distribution and reproduction in any medium or format, as long as you give appropriate credit to the original author(s) and the source, provide a link to the Creative Commons licence, and indicate if changes were made. The images or other third party material in this article are included in the article's Creative Commons licence, unless indicated otherwise in a credit line to the material. If material is not included in the article's Creative Commons licence and your intended use is not permitted by statutory regulation or exceeds the permitted use, you will need to obtain permission directly from the copyright holder. To view a copy of this licence, visit http://creativecommons.org/licenses/by/4.0/.

\section{References}

1. Barreiro-Perez M, Curione D, Symons R, Claus P, Voigt JU, Bogaert J (2018) Left ventricular global myocardial strain assessment comparing the reproducibility of four commercially available CMR-feature tracking algorithms. Eur Radiol 28:5137-5147

2. Plana JC, Galderisi M, Barac A et al (2014) Expert consensus for multimodality imaging evaluation of adult patients during and after cancer therapy: a report from the American Society of Echocardiography and the European Association of Cardiovascular Imaging. Eur Heart J Cardiovasc Imaging 15: 1063-1093

3. Celutkiene J, Plymen CM, Flachskampf FA et al (2018) Innovative imaging methods in heart failure: a shifting paradigm in cardiac assessment. Position statement on behalf of the Heart Failure Association of the European Society of Cardiology. Eur J Heart Fail 20:1615-1633

4. Schuster A, Paul M, Bettencourt N et al (2013) Cardiovascular magnetic resonance myocardial feature tracking for quantitative viability assessment in ischemic cardiomyopathy. Int $\mathrm{J}$ Cardiol 166:413-420

5. Buss SJ, Emami M, Mereles D et al (2012) Longitudinal left ventricular function for prediction of survival in systemic light-chain amyloidosis: incremental value compared with clinical and biochemical markers. J Am Coll Cardiol 60:1067-1076

6. Kraigher-Krainer E, Shah AM, Gupta DK et al (2014) Impaired systolic function by strain imaging in heart failure with preserved ejection fraction. J Am Coll Cardiol 63:447-456

7. Mangion K, Carrick D, Carberry J et al (2019) Circumferential strain predicts major adverse cardiovascular events following an acute ST-segment-elevation myocardial infarction. Radiology 290:329-337

8. Maret E, Todt T, Brudin L et al (2009) Functional measurements based on feature tracking of cine magnetic resonance images identify left ventricular segments with myocardial scar. Cardiovasc Ultrasound 7:53

9. Mor-Avi V, Lang RM, Badano LP et al (2011) Current and evolving echocardiographic techniques for the quantitative evaluation of cardiac mechanics: ASE/EAE consensus statement on methodology and indications endorsed by the Japanese Society of Echocardiography. Eur J Echocardiogr 12:167-205

10. Cho GY, Marwick TH, Kim HS, Kim MK, Hong KS, Oh DJ (2009) Global 2-dimensional strain as a new prognosticator in patients with heart failure. J Am Coll Cardiol 54:618-624
11. Illman JE, Arunachalam SP, Arani A et al (2018) MRI feature tracking strain is prognostic for all-cause mortality in AL amyloidosis. Amyloid 25:101-108

12. Reisner SA, Lysyansky P, Agmon Y, Mutlak D, Lessick J, Friedman Z (2004) Global longitudinal strain: a novel index of left ventricular systolic function. J Am Soc Echocardiogr 17:630-633

13. Romano S, Judd RM, Kim RJ et al (2018) Feature-tracking global longitudinal strain predicts death in a multicenter population of patients with ischemic and nonischemic dilated cardiomyopathy incremental to ejection fraction and late gadolinium enhancement. JACC Cardiovasc Imaging 11:1419-1429

14. Vigneault DM, Yang E, Jensen PJ et al (2019) Left ventricular strain is abnormal in preclinical and overt hypertrophic cardiomyopathy: cardiac MR feature tracking. Radiology 290:640-648

15. Voigt JU, Pedrizzetti G, Lysyansky P et al (2015) Definitions for a common standard for 2D speckle tracking echocardiography: consensus document of the EACVI/ASE/Industry Task Force to standardize deformation imaging. Eur Heart J Cardiovasc Imaging 16: $1-11$

16. Pedrizzetti G, Claus P, Kilner PJ, Nagel E (2016) Principles of cardiovascular magnetic resonance feature tracking and echocardiographic speckle tracking for informed clinical use. J Cardiovasc Magn Reson 18:51

17. Neizel M, Lossnitzer D, Korosoglou G et al (2009) Strain-encoded (SENC) magnetic resonance imaging to evaluate regional heterogeneity of myocardial strain in healthy volunteers: comparison with conventional tagging. J Magn Reson Imaging 29:99-105

18. Osman NF, Sampath S, Atalar E, Prince JL (2001) Imaging longitudinal cardiac strain on short-axis images using strain-encoded MRI. Magn Reson Med 46:324-334

19. Aletras AH, Ding S, Balaban RS, Wen H (1999) DENSE: displacement encoding with stimulated echoes in cardiac functional MRI. J Magn Reson 137:247-252

20. Cao JJ, Ngai N, Duncanson L, Cheng J, Gliganic K, Chen Q (2018) A comparison of both DENSE and feature tracking techniques with tagging for the cardiovascular magnetic resonance assessment of myocardial strain. J Cardiovasc Magn Reson 20:26

21. Zerhouni EA, Parish DM, Rogers WJ, Yang A, Shapiro EP (1988) Human heart: tagging with MR imaging-a method for noninvasive assessment of myocardial motion. Radiology 169:59-63

22. Altiok E, Neizel M, Tiemann S et al (2013) Layer-specific analysis of myocardial deformation for assessment of infarct transmurality: comparison of strain-encoded cardiovascular magnetic resonance with 2D speckle tracking echocardiography. Eur Heart J Cardiovasc Imaging 14:570-578

23. Hor KN, Baumann R, Pedrizzetti G et al (2011) Magnetic resonance derived myocardial strain assessment using feature tracking. J Vis Exp. https://doi.org/10.3791/2356

24. Blaszczyk E, Topper A, Schmacht L et al (2017) Influence of spatial resolution and contrast agent dosage on myocardial T1 relaxation times. MAGMA 30:85-91

25. Schmacht L, Traber J, Grieben U et al (2016) Cardiac involvement in myotonic dystrophy type 2 patients with preserved ejection fraction: detection by cardiovascular magnetic resonance. Circ Cardiovasc Imaging 9(7):e004615. https://doi.org/10.1161/ CIRCIMAGING.115.004615

26. von Knobelsdorff-Brenkenhoff F, Prothmann M, Dieringer MA et al (2013) Myocardial T1 and T2 mapping at $3 \mathrm{~T}$ : reference values, influencing factors and implications. J Cardiovasc Magn Reson 15:53

27. von Knobelsdorff-Brenkenhoff F, Schuler J, Doganguzel S et al (2017) Detection and monitoring of acute myocarditis applying quantitative cardiovascular magnetic resonance. Circ Cardiovasc Imaging 10(2):e005242. https://doi.org/10.1161/CIRCIMAGING. 116.005242 
28. Wassmuth R, Prothmann M, Utz W et al (2013) Variability and homogeneity of cardiovascular magnetic resonance myocardial T2mapping in volunteers compared to patients with edema. J Cardiovasc Magn Reson 15:27

29. Funk S, Kermer J, Doganguezel S, Schwenke C, von KnobelsdorffBrenkenhoff F, Schulz-Menger J (2018) Quantification of the left atrium applying cardiovascular magnetic resonance in clinical routine. Scand Cardiovasc J 52:85-92

30. Schulz-Menger J, Bluemke DA, Bremerich J et al (2020) Standardized image interpretation and post-processing in cardiovascular magnetic resonance - 2020 update: Society for Cardiovascular Magnetic Resonance (SCMR): board of trustees task force on standardized post-processing. J Cardiovasc Magn Reson 22:19

31. Cerqueira MD, Weissman NJ, Dilsizian V et al (2002) Standardized myocardial segmentation and nomenclature for tomographic imaging of the heart. A statement for healthcare professionals from the Cardiac Imaging Committee of the Council on Clinical Cardiology of the American Heart Association. Circulation 105:539-542

32. Schuster A, Morton G, Hussain ST et al (2013) The intra-observer reproducibility of cardiovascular magnetic resonance myocardial feature tracking strain assessment is independent of field strength. Eur J Radiol 82:296-301

33. Morton G, Schuster A, Jogiya R, Kutty S, Beerbaum P, Nagel E (2012) Inter-study reproducibility of cardiovascular magnetic resonance myocardial feature tracking. J Cardiovasc Magn Reson 14:43

34. Maceira AM, Tuset-Sanchis L, Lopez-Garrido M et al (2018) Feasibility and reproducibility of feature-tracking-based strain and strain rate measures of the left ventricle in different diseases and genders. J Magn Reson Imaging 47:1415-1425

35. Gertz RJ, Lange T, Kowallick JT et al (2018) Inter-vendor reproducibility of left and right ventricular cardiovascular magnetic resonance myocardial feature-tracking. PLoS One 13:e0193746

36. Augustine D, Lewandowski AJ, Lazdam M et al (2013) Global and regional left ventricular myocardial deformation measures by magnetic resonance feature tracking in healthy volunteers: comparison with tagging and relevance of gender. J Cardiovasc Magn Reson $15: 8$

37. Farsalinos KE, Daraban AM, Unlu S, Thomas JD, Badano LP, Voigt JU (2015) Head-to-head comparison of global longitudinal strain measurements among nine different vendors: the EACVI/ ASE inter-vendor comparison study. J Am Soc Echocardiogr 28(1171-1181):e1172

38. Liu B, Dardeer AM, Moody WE et al (2018) Reference ranges for three-dimensional feature tracking cardiac magnetic resonance: comparison with two-dimensional methodology and relevance of age and gender. Int J Cardiovasc Imaging 34:761-775

39. Taylor RJ, Moody WE, Umar F et al (2015) Myocardial strain measurement with feature-tracking cardiovascular magnetic resonance: normal values. Eur Heart J Cardiovasc Imaging 16:871-881

40. Andre F, Steen H, Matheis P et al (2015) Age- and gender-related normal left ventricular deformation assessed by cardiovascular magnetic resonance feature tracking. J Cardiovasc Magn Reson $17: 25$

41. Peng J, Zhao X, Zhao L et al (2018) Normal values of myocardial deformation assessed by cardiovascular magnetic resonance feature tracking in a healthy Chinese population: a multicenter study. Front Physiol 9:1181

42. Mangion K, Burke NMM, McComb C, Carrick D, Woodward R, Berry C (2019) Feature-tracking myocardial strain in healthy adults- a magnetic resonance study at 3.0 tesla. Sci Rep 9:3239

43. Backhaus SJ, Metschies G, Billing M et al (2019) Cardiovascular magnetic resonance imaging feature tracking: impact of training on observer performance and reproducibility. PLoS One 14:e0210127

44. Feisst A, Kuetting DLR, Dabir D et al (2018) Influence of observer experience on cardiac magnetic resonance strain measurements using feature tracking and conventional tagging. Int J Cardiol Heart Vasc 18:46-51

Publisher's note Springer Nature remains neutral with regard to jurisdictional claims in published maps and institutional affiliations. 